\title{
Síntese de pigmento cerâmico verde com base na estrutura cristalina da wollastonita
}

\section{Synthesis of ceramic green pigment based on the wollastonite crystal structure}

Gabriel Beck de Albuquerque ${ }^{1}$, Thais de Jesus Schimitt Ballmann ${ }^{1}$, Marilena Valadares Folgueras ${ }^{1}$, Sonia Richartz Prim ${ }^{2}$

\footnotetext{
${ }^{1}$ Programa de Pós Graduação em Ciência e Engenharia de Materiais - Universidade do Estado de Santa Catarina - Departamento de Engenharia Mecânica. PGCEM/UDESC/DEM CEP 89219-710 Joinville, SC

e-mail: marilena.folgueras@udesc.br

${ }^{2}$ Universidade Católica de Santa Catarina - Joinville - SC

e-mail: sorprim@gmail.com
}

\section{RESUMO}

Na indústria cerâmica de revestimento a cor é um parâmetro de grande importância no desenvolvimento de produtos. $\mathrm{O}$ aspecto visual da peça cerâmica e seu valor de mercado são influenciados por parâmetros como tonalidade, brilho e intensidade de cor. Neste segmento, a busca de novos pigmentos é constante, com foco na redução de custos, na adequação ambiental de pigmentos e na necessidade de inovação em cores e processos. Estes pigmentos, quando dispersos em um sólido, devem ser capazes de desenvolver cor mantendo-se insolúveis e inertes nas elevadas temperaturas de processamento. O objetivo do presente trabalho foi a síntese de pigmento cerâmico com a estrutura de Wollastonita - $\mathrm{CaSiO}_{3}$, com a inclusão de cromo como íon cromóforo, em teores variados. A difratometria de raios-X foi utilizada para avaliar o efeito da adição do cromo na formação das fases cristalinas. A análise de distribuição de tamanho de partícula foi realizada pela técnica de difração a laser. A análise colorimétrica foi realizado pela incorporação do pigmento em esmalte comercial e a avaliação da cor desenvolvida, através da determinação dos parâmetros colorimétricos L ${ }^{*}, a^{*}, b^{*}$. Os resultados mostraram que o teor de cromo utilizado e a temperatura de síntese interferem no desenvolvimento dos silicatos e na performance dos pigmentos.

Palavras-chave: Pigmentos cerâmicos, Wollastonita.

\begin{abstract}
In the ceramic industry, color is of great importance to product development. Parameters such as hue, brightness and color intensity influence the visual appearance of the ceramic surface and its market value. Research on the development of new pigments is ongoing, with focus on cost reduction, environmental suitability and color innovation. These pigments, when dispersed in a solid must be capable of developing color by keeping insoluble and inert at high processing temperatures. This study investigates the synthesis of ceramic pigment with the structure of wollastonite $-\mathrm{CaSiO}_{3}$, with the use of chromium at different ratios as chromophore ion. It was used X-ray diffraction in order to evaluate the effect of adding chromium to the formation of crystalline phases. Particle size analysis was performed by Laser diffraction technique and a colorimetric study was conducted through the analysis of the pigment applied to a commercial enamel, by determining colorimetric parameters $L^{*}, a^{*}$ and $b^{*}$. The results showed that chromium content and synthesis temperature interferes with the development of silicates and with the performance of new pigments.
\end{abstract}

Keywords: Ceramic Pigments, Wollastonite.

\section{INTRODUÇÃO}

Os pigmentos são materiais de grande importância em diferentes setores produtivos. Na indústria cerâmica não é diferente, principalmente nos segmentos onde fatores estéticos tem grande importância na qualidade do produto. Nestes setores, procura-se sempre o desenvolvimento de novos pigmentos que sejam eficientes, am- 
bientalmente corretos, inovadores e economicamente viáveis. Os pigmentos, muitas vezes, são utilizados em combinação com esmaltes e agentes opacificadores. Os esmaltes são materiais de natureza vítrea preparados por fusão de minerais industriais, cujo constituinte principal é o feldspato [1], enquanto os opacificadores são fases cristalinas, como Zirconita $\left(\mathrm{ZrSiO}_{4}\right)$, e óxidos de zircônio $\left(\mathrm{ZrO}_{2}\right)$, de zinco, $(\mathrm{ZnO})$, de titânio $\left(\mathrm{TiO}_{2}\right)$ e de estanho $\left(\mathrm{SnO}_{2}\right)$, que apresentam diferente índice de refração se comparado com o esmalte [2].

O pigmento cerâmico é formado, em geral, por óxidos complexos de metais de transição obtidos por um processo de calcinação. Estes materiais devem apresentar estabilidade térmica quando aquecidos, estabilidade química quando em contato com o esmalte ou a matriz cerâmica e boa eficiência pigmentante [3]. Deve-se considerar também que o pigmento necessita ser facilmente disperso na matriz, não pode promover a formação de voláteis que resultem em defeitos e deve apresentar propriedades óticas favoráveis, incluindo elevado índice de refração que garantirá a opacidade do material pigmentado [3][4].

Do ponto de vista químico, os pigmentos produzem cor pela ação de um íon cromóforo, em geral na forma de metais de transição, que absorve radiação visível de forma seletiva e é estabilizado por mecanismos químicos apropriados para conseguir manter sua ação pigmentante sob condições químicas e de temperatura desfavoráveis [5].

Para a síntese do pigmento, podem ser empregados em diferentes processos, sendo o mais utilizado industrialmente o método cerâmico convencional. Este método baseia-se na mistura de óxidos e tratamento térmico, geralmente em temperaturas elevadas. É um processo relativamente barato, porém o lento processo de difusão e as elevadas temperaturas de reação utilizadas são algumas das desvantagens. Além disso, tornase difícil a obtenção de pós monofásicos em escala micrométrica $(0,1-10 \mu \mathrm{m})[6]$.

Pigmentos formados com base na estrutura de silicatos são comuns e estes são formados com base na estrutura de zirconita, Forsterita, Mervinita, Diopsitas, Wellemita, Wollastonita, entre outros [7] [8] [9][10][11]. Estes pigmentos são obtidos a partir de matérias primas ricas em silício, cálcio, magnésio e óxidos fonte de íons cromóforos como Cobalto, Níquel, Cromo e Vanádio[11]. Entre as matérias primas empregadas podem ser consideradas matérias primas comerciais na forma de reagentes de elevada pureza, minérios [12] ou mesmo subprodutos agroindustriais [13] e industriais[14].

O presente trabalho tem por objetivo verificar a viabilidade de síntese de pigmento cerâmico, com base na estrutura cristalina da wollastonita, para aplicação em esmalte. Como matérias-primas foram utilizados reagentes de uso comercial e processo de reação no estado sólido em alta temperatura. Na elaboração do método descartou-se o uso de agentes mineralizadores de forma a desenvolver um processo em conformidade com a tendência de desenvolvimento de processos ambientalmente corretos.

\section{MATERIAIS E MÉTODOS}

Para a síntese dos pigmentos foram empregadas matérias primas comerciais, na forma de carbonato de cálcio (Vetec - grau de pureza 97,5\% em peso), sílica na forma de quartzo (Endeka- grau de pureza 98,9\% em peso) e óxido de cromo (Biotec- grau de pureza $98,0 \%$ em peso).

$\mathrm{Na}$ formulação, considerou-se como base a composição estequiométrica da wollastonita $\left(\mathrm{CaO} \cdot \mathrm{SiO}_{2}\right)$, com substituição parcial do íon cálcio $\left(\mathrm{Ca}^{2+}\right)$ por íon cromo $\left(\mathrm{Cr}^{3+}\right)$. Os cálculos estequiométricos relativos à massa dos reagentes utilizados para o preparo dos pigmentos foram realizados respeitando-se a razão equimolar entre reagentes e produtos, de forma a obter a massa total de $10 \mathrm{~g}$ de material. Na formulação foi considerada a formação de $\mathrm{Ca}_{6-\mathrm{x}} \mathrm{Cr}_{\mathrm{y}}\left(\mathrm{Si}_{2} \mathrm{O}_{6}\right)_{3}$, onde assumiram-se os valores de $\mathrm{x}$ e y apresentados na Tabela 1 .

Tabela 1: Identificação das formulações desenvolvidas para a síntese dos pigmentos

\begin{tabular}{c|c|c|c}
\hline $\mathbf{X}$ & $\mathbf{Y}$ & Denominação & Código \\
\hline 0 & 0,00 & $\mathrm{Ca}_{6}\left(\mathrm{Si}_{2} \mathrm{O}_{6}\right)_{3}$ & $\mathrm{CaCr}_{0}$ \\
\hline 1 & 0,67 & $\mathrm{Ca}_{5} \mathrm{Cr}_{0,67}\left(\mathrm{Si}_{2} \mathrm{O}_{6}\right)_{3}$ & $\mathrm{CaCr}_{0,67}$ \\
\hline 2 & 1,34 & $\mathrm{Ca}_{4} \mathrm{Cr}_{1,34}\left(\mathrm{Si}_{2} \mathrm{O}_{6}\right)_{3}$ & $\mathrm{CaCr}_{1,34}$ \\
\hline 3 & 2,00 & $\mathrm{Ca}_{3} \mathrm{Cr}_{2}\left(\mathrm{Si}_{2} \mathrm{O}_{6}\right)_{3}$ & $\mathrm{CaCr}_{2,00}$ \\
\hline 4 & 2,67 & $\mathrm{Ca}_{2} \mathrm{Cr}_{2,67}\left(\mathrm{Si}_{2} \mathrm{O}_{6}\right)_{3}$ & $\mathrm{CaCr}_{2,67}$ \\
\hline 5 & 3,34 & $\mathrm{Ca}_{1} \mathrm{Cr}_{3,34}\left(\mathrm{Si}_{2} \mathrm{O}_{6}\right)_{3}$ & $\mathrm{CaCr}_{3,34}$ \\
\hline 6 & 4,00 & $\mathrm{Cr}_{4}\left(\mathrm{Si}_{2} \mathrm{O}_{6}\right)_{3}$ & $\mathrm{CaCr}_{4,00}$ \\
\hline
\end{tabular}


Para a síntese do pigmento foi utilizado o processo convencional que considera a mistura das matérias primas, seguida de calcinação em atmosfera oxidante. Para a mistura e moagem das matérias primas foi utilizada a moagem a úmido em moinho de bolas convencional. Foram empregadas esferas de alumina como agente de moagem e álcool etílico para formar a suspensão. Após a moagem, o material foi seco utilizando lâmpadas incandescentes com emissão de radiação infravermelha. A calcinação foi realizada em forno mufla, nas temperaturas de $1000^{\circ} \mathrm{C}, 1150^{\circ} \mathrm{C}$ e $1250^{\circ} \mathrm{C}$. Foi utilizada taxa de aquecimento de $5^{\circ} \mathrm{C} /$ minuto e patamar único de 3 horas na temperatura máxima.

Após a calcinação, o material foi desaglomerado manualmente empregando-se almofariz de ágata até obter 100\% passante em peneira de malha 250 mesh. Os pigmentos obtidos foram caracterizados por difratometria de raios-x, para identificar as fases cristalinas formadas; por microscopia eletrônica de varredura e análise granulométrica a laser, para a avaliação morfológica e dimensional das partículas. A difratometria de raios-x foi realizada em equipamento Shimadzu DRX6000, com tubo de cobre e velocidade de varredura de 2 graus por minuto. As micrografias foram obtidas em microscópio eletrônico de varredura marca JEOL JSM6701. A análise granulométrica foi realizada em analisador de tamanho de partícula a laser marca Shimadzu, modelo SALD2201.

Para a análise colorimétrica, do pigmento aplicado em revestimentos cerâmicos, foi utilizado colorímetro Technidyne, Color Touch2, Model ISO, nos padrões CIELAB (iluminante D65 num ângulo de $2^{\circ}$ ). Os resultados foram apresentados através das coordenadas $\mathrm{L}^{*}, \mathrm{a}^{*}, \mathrm{~b}^{*}$. O termo $\mathrm{L}^{*}$ representa a luminosidade em escala de 0 a 100, sendo que o valor zero corresponde ao preto e o valor 100 corresponde ao branco. $O$ termo $a^{*}$ representa o intervalo entre as cores verde (valores negativos) e vermelho (valores positivos). O termo $b^{*}$ representa o intervalo entre as cores azul (valores negativos) e amarelo (valores positivos).

A aplicação do pigmento foi feita sobre base cerâmica crua e engobada. O pigmento foi diluído em esmalte convencional, considerando o uso de $2 \%$ em peso de pigmento, e aplicado utilizando binil com abertura de $0,7 \mathrm{~mm}$. A queima foi feita utilizando forno de laboratório com ciclo térmico típico de processo de monoqueima e temperatura máxima de $1080^{\circ} \mathrm{C}$.

\section{RESULTADOS}

A difratometria de raios-X foi empregada como principal ferramenta para avaliar a formação de fases cristalinas específicas a partir da combinação das matérias primas selecionadas e tratamentos térmicos em diferentes temperaturas. Os difratogramas obtidos para os materiais submetidos a tratamento térmico na temperatura de $1000^{\circ} \mathrm{C}, 1150^{\circ} \mathrm{C}$ e $125^{\circ} \mathrm{C}$ estão representados nas Figuras 1,2 e 3 respectivamente.

Todos os materiais obtidos por tratamento térmico a 1150 e $1250^{\circ} \mathrm{C}$, em que ocorreu a formação de silicatos, foram submetidos a processo de moagem em condições similares, até atingir $100 \%$ do material passante em malha 250 mesh. Os resultados obtidos por meio da análise da distribuição do tamanho de partícula estão apresentados na Tabela 2 e mostram a influência da temperatura de síntese e da composição na eficiência de moagem do material.

As imagens obtidas por Microscopia Eletrônica de Varredura (MEV) em diferentes ampliações são mostradas nas Figuras 4 e 5. A Figura 4 apresenta os pigmentos, das formulações $\mathrm{CaCr}_{0,67}, \mathrm{CaCr}_{2,00} \mathrm{e} \mathrm{CaCr}$ 2,67, calcinados a $1150^{\circ} \mathrm{C}$. Na Figura 5 são apresentadas imagens comparativas do pigmento $\mathrm{CaCr}_{2,67}$ sintetizados a $1150^{\circ} \mathrm{C}$ e $1250^{\circ} \mathrm{C}$. As imagens permitem a observação da morfologia e tamanho de partículas presentes nos aglomerados formados, assim como a formação de fase vítrea para o material calcinado a $1250^{\circ} \mathrm{C}$.

Do ponto de vista de coloração, os pigmentos foram avaliados por análise visual, evidenciando o efeito do teor de $\mathrm{Cr}_{2} \mathrm{O}_{3}$ adicionado na coloração do pigmento formado.(Figura 6).

Para a avaliação do desempenho pigmentante dos materiais desenvolvidos, foram realizados testes usando como base um esmalte comercial e temperatura de queima de $1080^{\circ} \mathrm{C}$. Após a aplicação, foram determinadas as coordenadas colorimétricas L, $\mathrm{a}^{*} \mathrm{e} \mathrm{b}^{*}$. Os resultados estão apresentados na Tabela 3 e mostram que a coordenada $b^{*}$ apresentou pouca variação, enquanto as coodenadas $L$ e a ${ }^{*}$ sofreram influência direta do teor de cromo utilizado. 


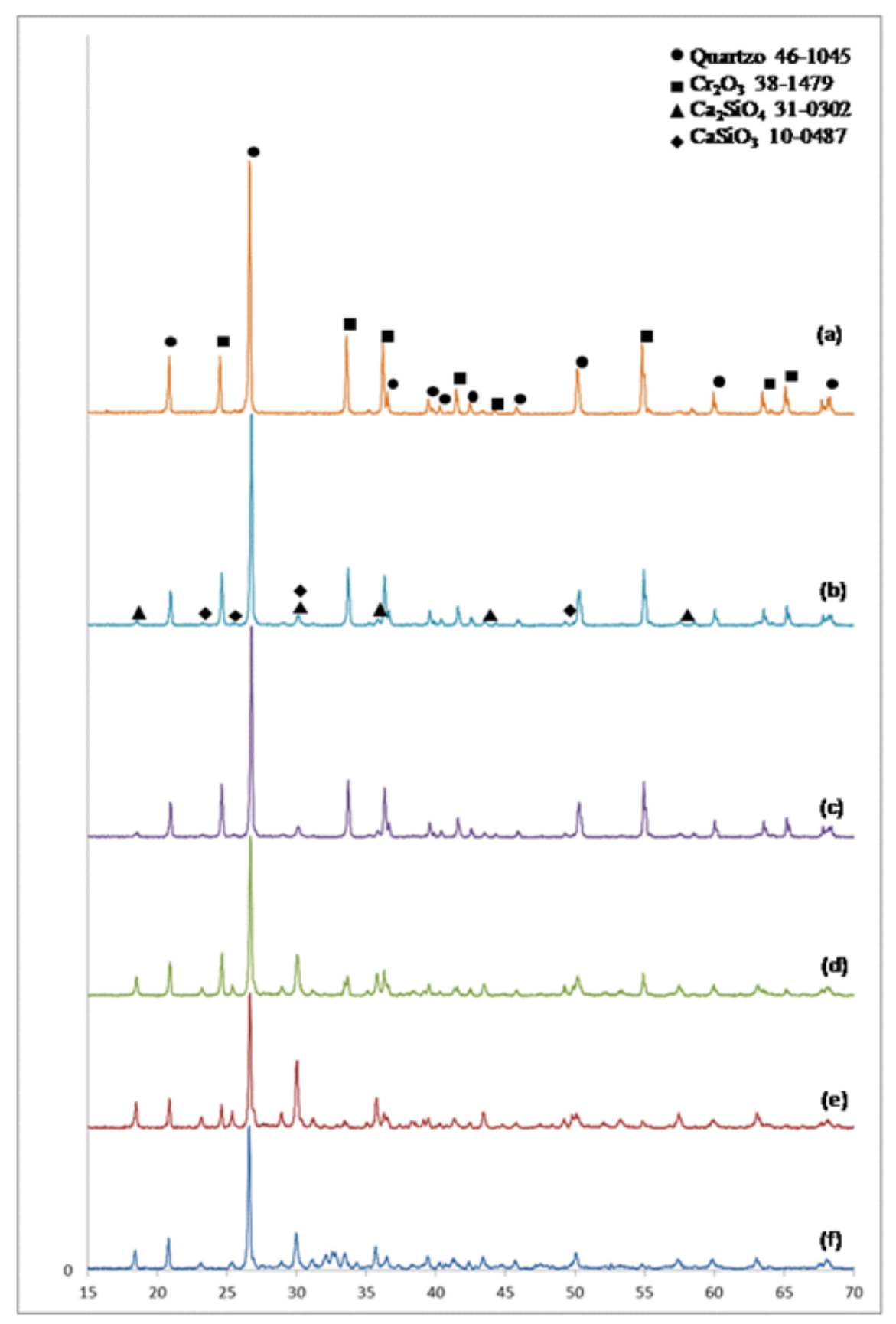

Figura 1: Difratograma de raios- $x$ das diferentes composições submetidas a tratamento térmico na temperatura de $1000^{\circ} \mathrm{C}$. (a) $\mathrm{Y}=4,00$; (b) $\mathrm{Y}=3,34$; (c) $\mathrm{Y}=2,67$; (d) $\mathrm{Y}=2,00$; (e) $\mathrm{Y}=1,34$; (f) $\mathrm{Y}=0,67$. 


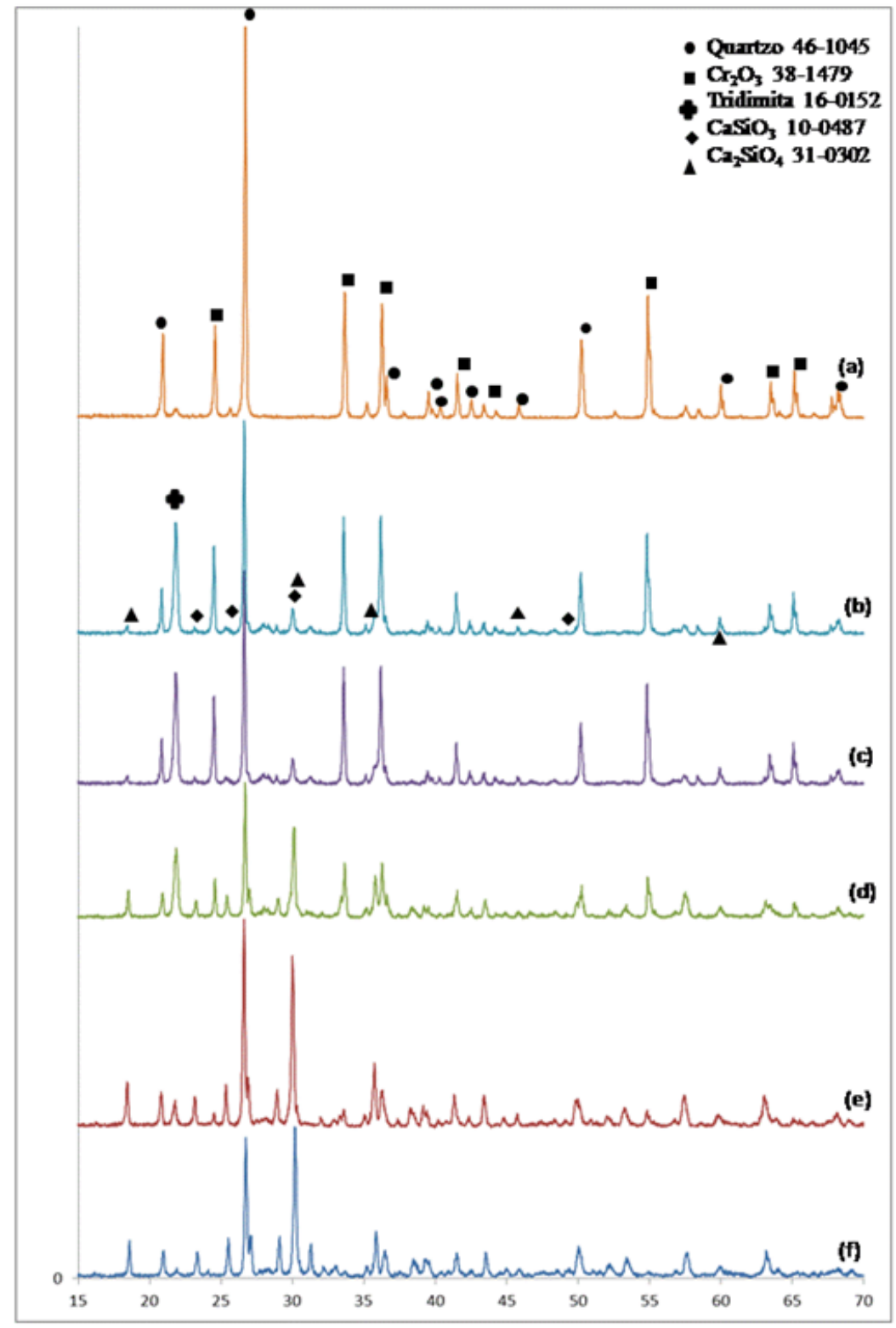

Figura 2: Difratograma de raios- $\mathrm{x}$ das diferentes composições submetidas a tratamento térmico na temperatura de $1150^{\circ} \mathrm{C}$. (a) $\mathrm{Y}=4,00$; (b) $\mathrm{Y}=3,34$; (c) $\mathrm{Y}=2,67$; (d) $\mathrm{Y}=2,00$; (e) $\mathrm{Y}=1,34$; (f) $\mathrm{Y}=0,67$. 


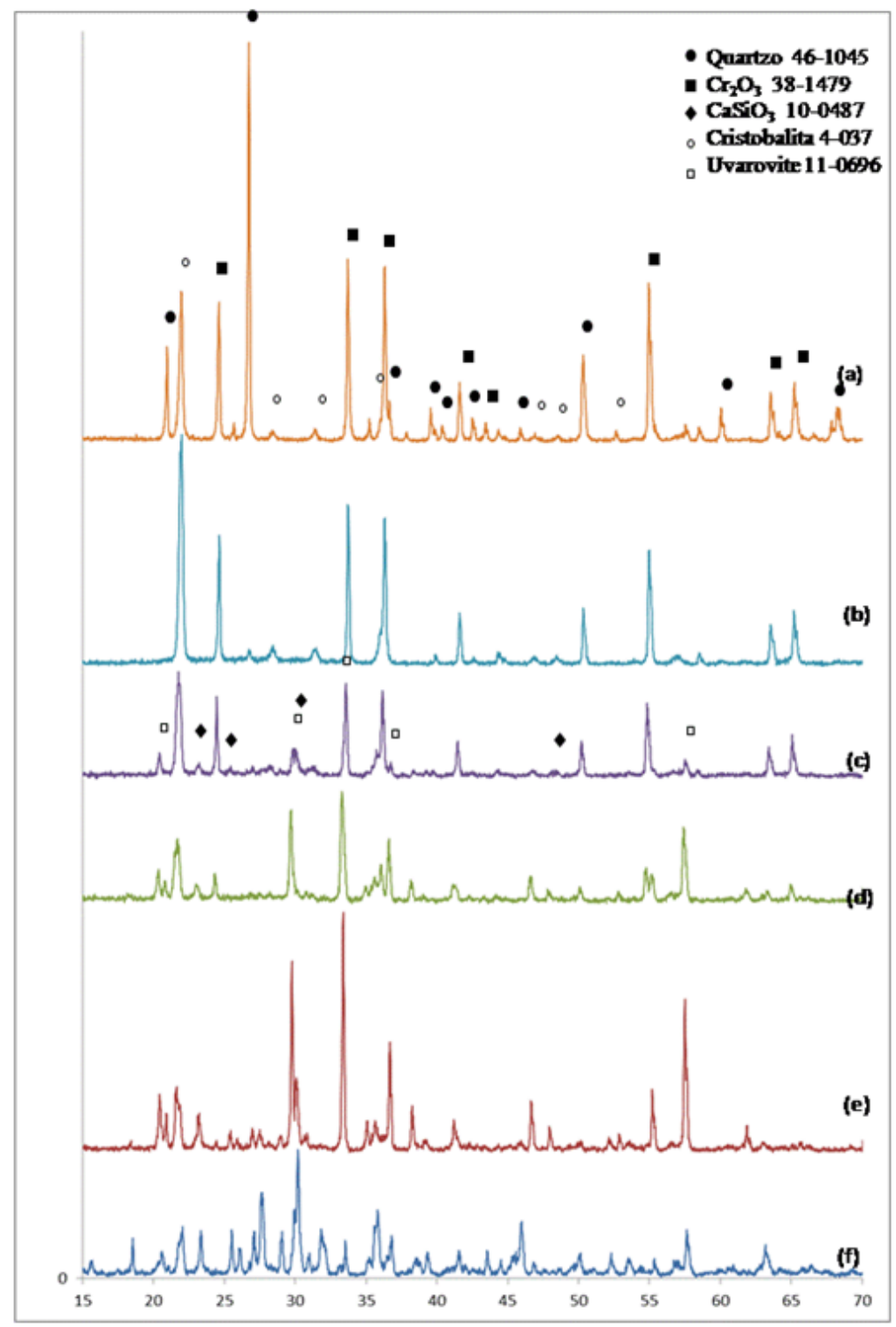

Figura 3: Difratograma de raios- $x$ das diferentes composições submetidas a tratamento térmico na temperatura de $1250^{\circ} \mathrm{C}$. (a) $\mathrm{Y}=4,00$; (b) $\mathrm{Y}=3,34$; (c) $\mathrm{Y}=2,67$; (d) $\mathrm{Y}=2,00$; (e) $\mathrm{Y}=1,34$; (f) $\mathrm{Y}=0,67$.

Tabela 2: Distribuição de tamanho de partículas do pigmento obtidos em diferentes temperaturas de síntese

\begin{tabular}{|c|c|c|c|c|}
\hline \multirow{2}{*}{ Temperatura $\left({ }^{\circ} \mathrm{C}\right)$} & & \multicolumn{3}{|c|}{ Diâmetro $(\mu \mathrm{m})<$} \\
\hline & & $\mathrm{CaCr} 0,00$ & $\mathrm{CaCr} 2,00$ & $\mathrm{CaCr} 2,67$ \\
\hline \multirow{5}{*}{1150} & $\mathrm{D}_{10}$ & 0,92 & 6,87 & 7,17 \\
\hline & $\mathrm{D}_{30}$ & 9,76 & 13,80 & 12,21 \\
\hline & $\mathrm{D}_{50}$ & 13,53 & 19,87 & 16,59 \\
\hline & $\mathrm{D}_{70}$ & 17,91 & 20,32 & 21,91 \\
\hline & $\mathrm{D}_{90}$ & 25,62 & 51,26 & 32,08 \\
\hline \multirow{5}{*}{1250} & $\mathrm{D}_{10}$ & 0,66 & 9,456 & 21,53 \\
\hline & $\mathrm{D}_{30}$ & 1,24 & 60,74 & 29,84 \\
\hline & $\mathrm{D}_{50}$ & 89,92 & 102,63 & 43,11 \\
\hline & $\mathrm{D}_{70}$ & 154,15 & 144,28 & 58,07 \\
\hline & $\mathrm{D}_{90}$ & 224,25 & 212,18 & 97,99 \\
\hline
\end{tabular}


ALBUQUERQUE, G.B.; BALLMAN, T.J.S.; FOLGUERAS, M.V.; PRIM, S.R. revista Matéria, v.21, n.2, pp. 355 - $364,2016$.
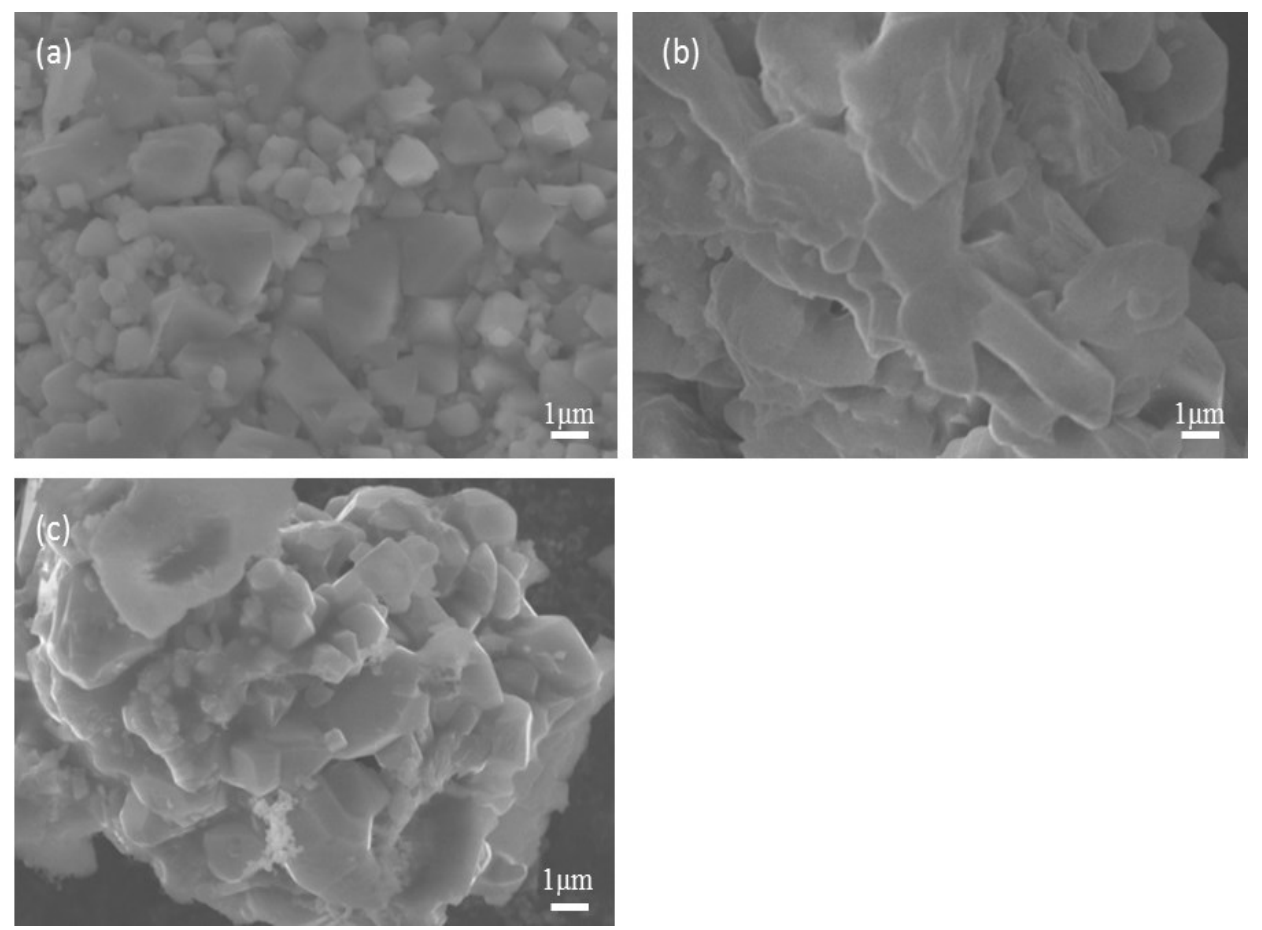

Figura 4: Micrografias representativa dos pigmentos sintetizados a $1150^{\circ} \mathrm{C}$, obtidas por microscopia eletrônica de varredura - Elétrons secundários. (a) $\mathrm{CaCr} 0,67$; (b) $\mathrm{CaCr}_{2,00}$; (c) $\mathrm{CaCr}_{2,67}$.
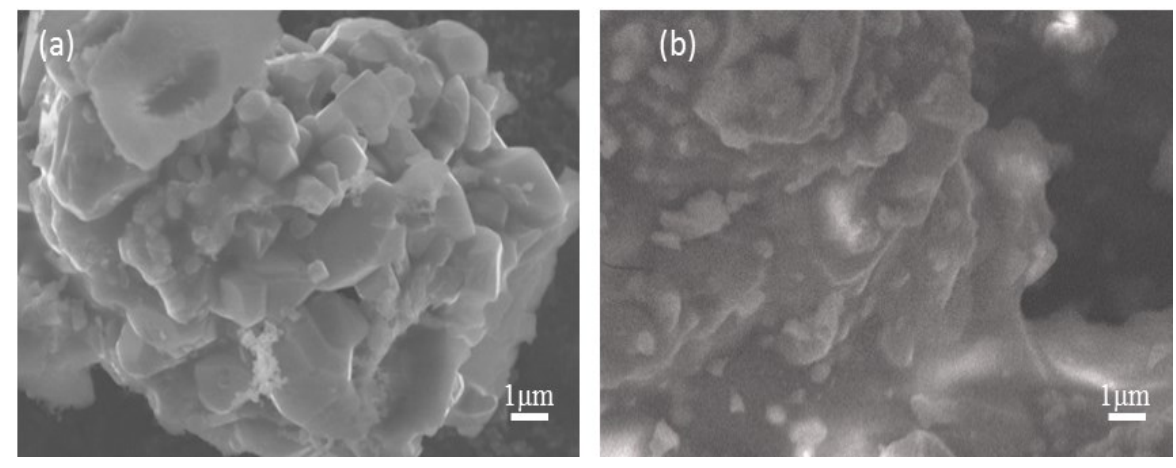

Figura 5: Micrografias representativas da morfologia, obtidas por microscopia eletrônica de varredura - Elétrons secundários, de partículas dos pigmentos $\mathrm{CaCr}_{2,6}$ sintetizados a (a) $1150^{\circ} \mathrm{C}$ e (b) $1250^{\circ} \mathrm{C}$ 


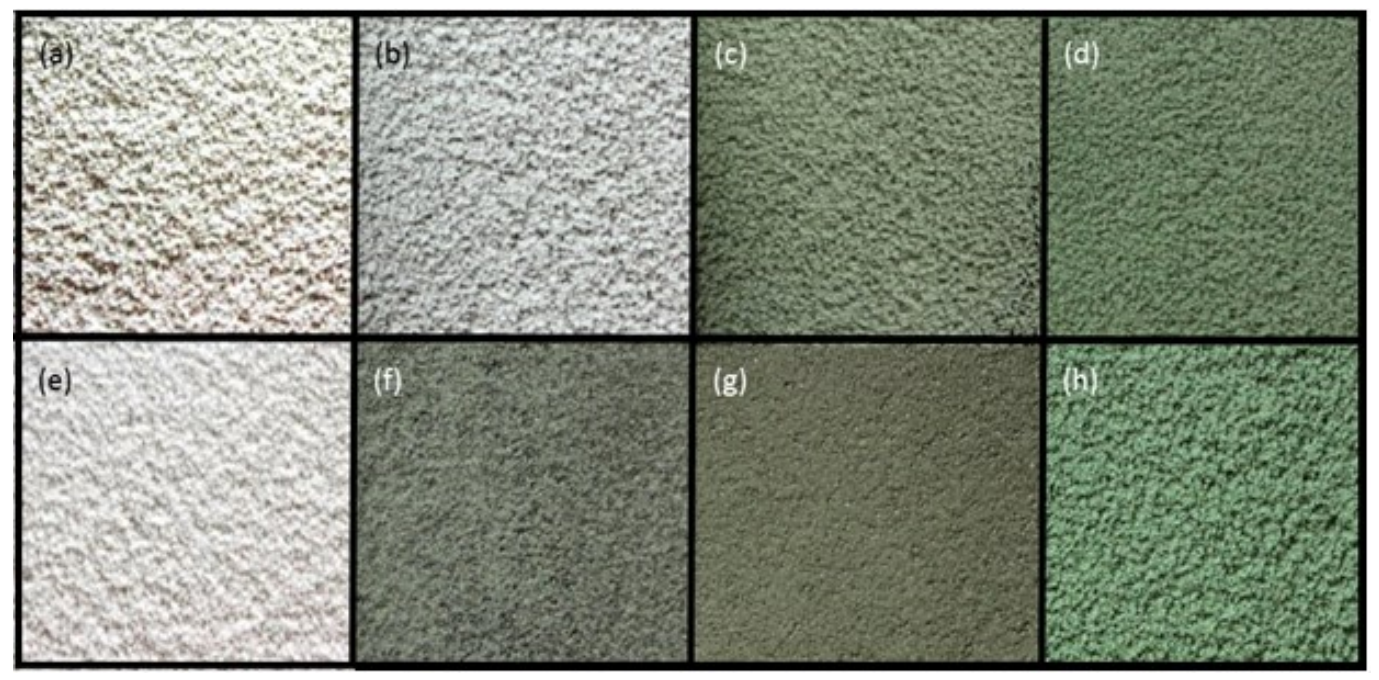

Figura 6: Imagem representativa dos pigmentos produzidos sintetizados a $1150^{\circ} \mathrm{C}$ (a) $\mathrm{CaCr}_{0}$, (b) $\mathrm{CaCr}_{1,34}(\mathrm{c}) \mathrm{CaCr}_{2,67}(\mathrm{~d})$ $\mathrm{CaCr}_{4,00}$ e $1250^{\circ} \mathrm{C}$ (e) $\mathrm{CaCr}_{0}$, (f) $\mathrm{CaCr}_{1,34}$ (g) $\mathrm{CaCr}_{2,67}$ (h) $\mathrm{CaCr}_{4,00}$

Tabela 3: Parâmetros colorimétricos para pigmentos sintetizados a $1150^{\circ} \mathrm{C}$ e $1250^{\circ} \mathrm{C}$, após aplicação em esmalte cerâmico e queima a $1080{ }^{\circ} \mathrm{C}$.

\begin{tabular}{l|ccc|ccc}
\hline \multirow{2}{*}{ Amostras } & \multicolumn{3}{|c|}{$1150^{\circ} \mathrm{C}$} & \multicolumn{3}{c}{$1250^{\circ} \mathrm{C}$} \\
\cline { 2 - 7 } & $\mathrm{L}$ & $\mathrm{a}^{*}$ & $\mathrm{~b}^{*}$ & $\mathrm{~L}$ & $\mathrm{a}^{*}$ & $\mathrm{~b}^{*}$ \\
$\mathrm{CaCr} 0,67$ & 68,91 & 1,14 & 14,20 & 68,73 & 0,29 & 12,81 \\
$\mathrm{CaCr} 1,34$ & 56,52 & $-2,77$ & 12,71 & 65,96 & $-1,23$ & 13,04 \\
$\mathrm{CaCr} 2,00$ & 50,05 & $-3,59$ & 11,26 & 61,05 & $-1,52$ & 11,6 \\
$\mathrm{CaCr} 2,67$ & 46,05 & $-6,99$ & 12,22 & $\mathrm{NR}^{+}$ & $\mathrm{NR}^{+}$ & $\mathrm{NR}^{+}$ \\
$\mathrm{CaCr} 3,34$ & 42,39 & $-11,71$ & 12,83 & 47,21 & $-6,14$ & 11,17 \\
$\mathrm{CaCr} 4,00$ & 38,92 & $-12,61$ & 12,84 & 39,04 & $-11,68$ & 13,45 \\
\hline
\end{tabular}

${ }^{+}$Não realizado.

\section{DISCUSSÃO}

A figura 1 apresenta os difratogramas obtidos para os materiais submetido a tratamento térmico na temperatura de $1000^{\circ} \mathrm{C}$. Foi possível identificar, por difratometria de raios-X, a presença de sílica na forma de quartzo (ICDD 46-1045) e óxido de cromo, na forma de $\mathrm{Cr}_{2} \mathrm{O}_{3}$ (ICDD 38-1479) para todas as composições (Figura 1). Observa-se, também, a formação de silicato de cálcio, na forma de $\mathrm{Ca}_{2} \mathrm{SiO}_{4}$ (ICDD 31-0302) e $\mathrm{CaSiO}_{3}$ (ICDD 10-0487), sendo a segunda fase denominada por Wollastonita. A formação do silicato é prejudicada pelo aumento do teor de óxido de cromo adicionado.

Como o aumento da temperatura de síntese do pigmento para $1150^{\circ} \mathrm{C}$, foi favorecida a formação de wollastonita (ICDD 10-0487) (ICDD 43-1460) (Figura 2). A formação do espectro de difração característico desta fase foi acompanhada da diminuição acentuada da intensidade relativa dos picos associados à presença do óxido de cromo $\mathrm{Cr}_{2} \mathrm{O}_{3}$ (ICDD 381479) e sílica.

$\mathrm{O}$ aumento do teor de óxido de cromo empregado na formulação inibiu a formação da wollastonita, indicando claramente a existência de um limite máximo de óxido de cromo a ser adicionado ao sistema para a formação dos silicatos de cálcio. Este efeito é semelhante ao observado para o material submetido a tratamento térmico em $1000^{\circ} \mathrm{C}$. Pode ser observado, também, que quando a sílica, adicionada na forma de quartzo, não é envolvida na formação do silicato de cálcio ocorre a conversão parcial desta fase em tridimita (ICDD 01-0378)

Com o aumento da temperatura de síntese do pigmento, para $1250^{\circ} \mathrm{C}$, foi mantida a tendência de comportamento em que teores maiores de óxido de cromo resultaram na inibição da formação de silicato de cál- 
cio (Figura 3). Nestes casos, foi identificada apenas a transformação de quartzo para tridimita ou cristobalita. Para composições contendo teores de óxido de cromo menores foi identificada a formação de silicatos de cálcio na forma de wollastonita e uvarovita. A formação de silicatos de cálcio foi acompanhada da redução da intensidade relativa dos picos característicos da presença de óxido de cromo.

Os resultados de distribuição do tamanho de partícula, apresentados na Tabela 2, mostraram que o aumento da temperatura de síntese do pigmento, de $1150^{\circ} \mathrm{C}$ para $1250^{\circ} \mathrm{C}$, resultou em um material com partículas grosseiras. Quando considerado o tratamento térmico a $1150^{\circ} \mathrm{C}$ não pode ser determinada uma relação direta entre o teor de cromo empregado e o tamanho médio de partícula (D50), sendo mantidos valores semelhantes para todas as composições. Já para o material submetido a tratamento térmico a $1250^{\circ} \mathrm{C}$ percebe-se uma variação do diâmetro médio de partícula (D50) com a variação do teor de óxido de cromo. O aumento do tamanho médio de partícula com a temperatura de tratamento térmico pode estar associado à formação de fase líquida que favoreceu o processo de sinterização entre as partículas que constituem o material, o que dificulta o processo de moagem.

A diferença granulométrica entre os materiais pode provocar variação na tonalidade dos pigmentos, visto que o desenvolvimento da cor depende do tamanho de partícula do pigmento e da estabilidade destas partículas no meio a ser pigmentado [2]. Existe um compromisso em que partículas grosseiras resultam em maior dificuldade de dispersão do pigmento, enquanto partículas finas são mais susceptíveis à decomposição [15].

O controle da distribuição de tamanho de partícula do pigmento pode ser realizado por meio do controle da etapa de moagem durante o processamento. Entretanto, o resultado aqui apresentado mostra claramente que o pigmento obtido por tratamento térmico a $1250^{\circ} \mathrm{C}$ exigirá um processo de moagem mais eficiente, o que pode ser associado ao efeito de sinterização e formação de agregados de maior resistência[16]. Deve-se destacar, também, que a possibilidade de formação de fase líquida pode ser considerada como um fator que favorece o processo de reação para formação das fases cristalinas desejadas, entretanto, pode ser um indicativo de instabilidade térmica para algumas fases cristalinas.

A avaliação morfológica do pigmento moído, realizada por microscopia eletrônica de varredura, evidenciou a formação de aglomerados com partículas de formatos e dimensões variáveis para todas as composições(Figuras 4 e 5). Nestas imagens é possível identificar a formação de fase líquida para o material sintetizado a $1250^{\circ} \mathrm{C}$, o que efetivamente resulta na formação de agregados mais rígidos e de difícil moagem.

Do ponto de vista de coloração, os pigmentos foram avaliados por análise visual. O que pode ser observado é que o aumento do teor de óxido de cromo na formulação resulta, efetivamente, no aumento da intensidade da coloração, independentemente da formação ou não dos silicatos (Figura 6).

Quando avaliado o efeito pigmentante dos diferentes materiais (Tabela 3), através das coordenadas colorimétricas definiu-se como ponto de destaque o predomínio de valores a* negativos e de $\mathrm{b}^{*}$ positivos, sendo que o parâmetro ${ }^{*}$ apresenta variação significativa em função do teor de cromo, enquanto o parâmetro $b^{*}$ permanece praticamente inalterado.

Valores de $a^{*}$ negativo são típicos de predominância de coloração verde e valores de $b^{*}$ sempre positivos indicam coloração amarela. Porém, como os valores dos parâmetros de $b^{*}$ estão próximos do eixo acromático do espaço de cor $\mathrm{La}^{*} \mathrm{~b}^{*}$, a influência da cor amarela no resultado final é pequena.

A análise do desempenho de íons cromóforos no desenvolvimento da cor mostrou que o mesmo íon pode favorecer a formação de diferentes cores e tons e que o uso de cromo resulta no desenvolvimento de coloração de verde a verde acinzentado[17].

Quando os valores de $\mathrm{a}^{*} \mathrm{e} \mathrm{b}^{*}$ aumentam e a posição se move para fora do centro, a saturação da cor aumenta. Observando a grande variação de $\mathrm{a}^{*}$, percebe-se uma relação diretamente proporcional entre o aumento da porcentagem em massa do óxido de cromo nas formulações com a diminuição dos valores do parâmetro $\mathrm{a}^{*}$. Quanto menor for o valor de $\mathrm{a}^{*}$, considerando o valor de $\mathrm{b}^{*}$ fixo, maior será a saturação do verde.

Os valores de L variam entre 68,91 e 38,92, não havendo diferença entre as temperaturas estudadas. Esta variação indicando uma diminuição de luminosidade (que varia de negro (0) ao branco (100)) .

\section{CONCLUSÕES}

A partir dos experimentos realizados, é possível concluir que a estrutura cristalina proposta para formação do pigmento pode ser obtida controlando-se a composição da mistura e a temperatura de síntese. $\mathrm{O}$ aumento da temperatura favorece a formação de silicatos de cálcio. Por sua vez, o aumento do teor de óxido de cromo também tem um efeito positivo na formação de wollastonita e uvarovite, desde que seja respeitado o limite máximo de adição. Adições de quantidades superiores de óxido de cromo tendem a evitar a formação de 
silicatos, mesmo em temperaturas maiores. De forma geral, o aumento do percentual em massa de óxido de cromo resultou em um pigmento que, após a aplicação, apresentou maior tonalidade e menor luminosidade do verde nas temperaturas de calcinação utilizadas. Este efeito pode ser associado, em parte, pela ação pigmentante do óxido de cromo.

$\mathrm{O}$ aumento da temperatura de síntese resultou em menor eficiência de moagem do pigmento, o que pode ser associado à formação de fase líquida e aumento da dureza dos agregados formados. A presença dessa fase líquida favorece o processo de síntese do pigmento, entretanto pode resultar em menor estabilidade do mesmo em temperaturas elevadas, refletindo também em instabilidade da cor.

\section{AGRADECIMENTOS}

Agradecimentos à UDESC, à CAPES e a FAPESC pelo apoio financeiro para o desenvolvimento deste trabalho.

\section{BIBLIOGRAFIA}

[1] SÁNCHES, E. "Matérias-primas para a fabricação de fritas e esmaltes cerâmicos", Cerâmica Industrial, v. 2, n. 3/4, pp. 32-40, 1997.

[2] BONDIOLI, F, MANFREDINI, T., OLIVEIRA, A.P.N. "Pigmentos inorgânicos: Projeto, produção e aplicação industrial”, Cerâmica Industrial, v.3, pp. 4-6, 1998.

[3] DA COST A, A. F., Sintese e caracterização de espinélios a base de Cu, Fe e Cr para pigmentos cerâmicos, Dissertação de M.Sc., CCET/UFRN, Natal, RN, Brasil, 2010.

[4] MONROS, G. Pigment, Ceramic. Encyclopedia of Color Science and Technology, Springer Science Business Media, New York, 2013.

[5] DELLA, V.P., JUNKES, J.A., KUNKN, I., et al., "Utilização do subproduto da recuperação metálica de escórias de aços inoxidáveis na síntese de pigmentos cerâmicos; caracterização da matéria prima", Revista Cerâmica, v. 51, n. 318, pp. 111-116, 2005

[6] MUÑOZ, R., et al., "Environmental Study of $\mathrm{Cr}_{2} \mathrm{O}_{3}-\mathrm{Al}_{2} \mathrm{O}_{3}$ Green Ceramic Pigment Synthesis", Journal of the European Ceramic Society, n.24, pp. 2087-2094, 2004.

[7] SCHABBACH, L.M., BONDIOLI, F., FREDEL, M.C. "Colouring of Opaque Ceramic Glaze with Zircon Pigments: Formulation With Simplified Juberlka-Munk Model”, Journal of the European Ceramic Society, v.31, n 5, pp. 659-664,2011.

[8] HADRI, M., AHAMDANE, H., RAGHNI, M.A.. "Sol Gel Synthesis of Forsterite, M-doped Forsterite Solid Solutions and their use as Ceramic Pigments", Journal of the European Ceramic Society, v. 35 pp $765-$ $777,2015$.

[9] MASLENNIKOVA, G. N., GVOZDEVAL, N.A. "Synthesis of Ceramic Pigments with Merwinite Crystal Structure", Glass and Ceramics, v.69, n. 9-10, pp 330-332, 2013

[10] SEDEL'NIKOVA, M.B., PROGREBENKOV, V.M., "Production of ceramic pigments with wollastonite and diopside structures using nepheline sludge", Glass and Ceramics, v. 64, n. 9-10, pp. 363-365, 2007

[11] MASLENNIKOVA, G. N., PISHCH, I. V., RADION, E. V., "Current Classification of Ceramic Silicate Pigmentsm (REVIEW)”, Glass and Ceramics, V.63, n. 9-10 pp.281-284, 2006.

[12] SEDEL'NIKOVA, M. B., et al. "Nonstoichiometric Reactions Producing Ceramic Pigments", Glass and Ceramics, v. 68, n 3-4, 2011.

[13] ANDREOLA, F., BARBIERI, L., BONDIOLI, F. “Agricultural Waste in the Synthesis of Coral Ceramic Pigment”, Dyes and Pigments, v. 94, pp 207-221, 2012.

[14] PRIM, S.R., FOLGUERAS, M.V., LIMA, M.A., "Synthesis and Characterization of Hematite Pigment Obtanined from a Stell Waste Industry”, Journal of Hzardous Materials, v. 192, pp. 1307-1313, 2011.

[15] MATTEUCCI, F., BOSCHI,A.O., et al. "Colour development of red perovskite pigment $\mathrm{Y}(\mathrm{Al}, \mathrm{Cr}) \mathrm{O} 3$ in various ceramic applications”, Advances in Applied Ceramics, v. 105, 2006

[16] RIVES, V."Development of a black ceramic pigment from non stoichiometric hydrotalcites", Journal of the European Ceramic Society, v. 32, pp 975-987, 2012.

[17] NIKOVA, M.B.S., et al. "Nonstoichimetric Reactions Producing Ceramic Pigments", Glass and Ceramics, v.68, n.3-4, 2011. 